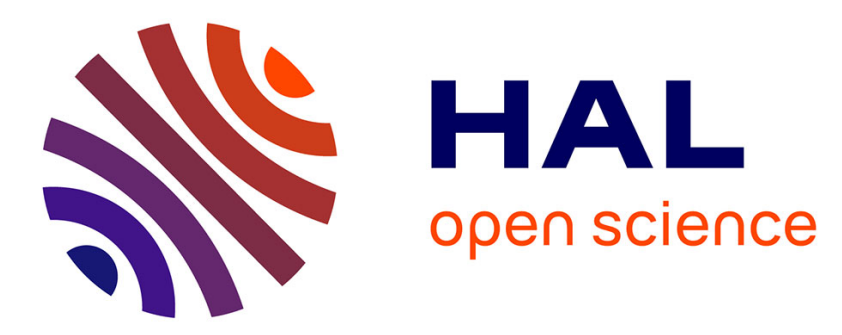

\title{
Primary resistance of hepatitis $B$ virus to nucleoside and nucleotide analogues
}

Stéphane Chevaliez, Christophe Rodriguez, Lila Poiteau, Alexandre Soulier, Flora Donati, Mélanie Darty-Mercier, Corinne Pioche, Vincent Leroy, Véronique Brodard, Fabien Zoulim, et al.

\section{To cite this version:}

Stéphane Chevaliez, Christophe Rodriguez, Lila Poiteau, Alexandre Soulier, Flora Donati, et al.. Primary resistance of hepatitis B virus to nucleoside and nucleotide analogues. Journal of Viral Hepatitis, 2019, 26 (2), pp.278-286. 10.1111/jvh.13025 . hal-03139196

\section{HAL Id: hal-03139196 https://hal.science/hal-03139196}

Submitted on 26 Apr 2021

HAL is a multi-disciplinary open access archive for the deposit and dissemination of scientific research documents, whether they are published or not. The documents may come from teaching and research institutions in France or abroad, or from public or private research centers.
L'archive ouverte pluridisciplinaire HAL, est destinée au dépôt et à la diffusion de documents scientifiques de niveau recherche, publiés ou non, émanant des établissements d'enseignement et de recherche français ou étrangers, des laboratoires publics ou privés. 


\title{
Primary resistance of hepatitis B virus to nucleoside and nucleotide analogues
}

\author{
Stéphane Chevaliez ${ }^{1,2}$ (i) | Christophe Rodriguez ${ }^{1,2}$ | Lila Poiteau ${ }^{1,2}$ | \\ Alexandre Soulier $^{1,2}$ | Flora Donati ${ }^{1,2}$ | Mélanie Darty-Mercier ${ }^{1,2}$ | Corinne Pioche ${ }^{3}$ | \\ Vincent Leroy $^{4,5}$ | Véronique Brodard ${ }^{6}$ | Fabien Zoulim ${ }^{7,8}$ (1) | Cécile Brouard ${ }^{3}$ | \\ Christine Larsen $^{3}$ | Caroline Semaille ${ }^{3}$ | Françoise Roudot-Thoraval ${ }^{9}$ | \\ Jean-Michel Pawlotsky ${ }^{1,2}$ | The Hepatology Reference Centers Laboratories Network for \\ Chronic Hepatitis B Surveillance*
}

\author{
${ }^{1}$ Department of Virology, Hôpital Henri \\ Mondor, National Reference Center for Viral \\ Hepatitis B, C and D, Université Paris-Est, \\ Créteil, France \\ ${ }^{2}$ INSERM U955, Créteil, France \\ ${ }^{3}$ Santé Publique France, Saint-Maurice, \\ France \\ ${ }^{4}$ Clinique d'Hépatogastroentérologie, Centre \\ Hospitalier Universitaire de Grenoble, \\ Grenoble, France \\ ${ }^{5}$ INSERM U823, Grenoble, France \\ ${ }^{6}$ Laboratoire de Virologie, Hôpital Robert \\ Debré, Reims, France \\ ${ }^{7}$ Service \\ d'Hépatogastroentérologie, Hospices Civils \\ de Lyon, Lyon, France \\ ${ }^{8}$ INSERM U1052, Cancer Research Center of \\ Lyon (CRCL), Lyon, France \\ ${ }^{9}$ Department of Public Health, Hôpital Henri \\ Mondor, Université Paris-Est, Créteil, France

\section{Correspondence} \\ Stéphane Chevaliez, Department of \\ Virology, Hôpital Henri Mondor, National \\ Reference Center for Viral Hepatitis B, C and \\ D, Université Paris-Est, Créteil, France. \\ Email: stephane.chevaliez@aphp.fr \\ Funding information \\ This work was supported by Santé publique \\ France. It was partly funded by the Agence \\ Nationale de Recherche sur le SIDA et \\ les Hépatites Virales (ANRS) and Gliead \\ Sciences.
}

\begin{abstract}
Summary
Nucleoside and nucleotide analogues (NUCs) targeting hepatitis B virus are capable of selecting resistant viruses upon long-term administration as monotherapies. The prevalence of resistance-associated substitutions (RASs) and fitness-associated substitutions at baseline of NUC therapy and their impact on treatment responses remain unknown. A total of 232 treatment-naïve patients chronically infected with hepatitis $B$ virus (HBV) consecutively referred for the first time to one of French reference centres were included. The nearly full-length HBV reverse transcriptase was sequenced by means of deep sequencing, and the sequences were analysed. RASs were detected in $25 \%$ of treatment-naïve patients, generally representing low proportions of the viral quasispecies. All amino acid positions known to be associated with HBV resistance to currently approved NUCs or with increased fitness of resistant variants were affected, except position 80. RASs at positions involved in lamivudine, telbivudine and adefovir resistance were the most frequently detected. All patients with RASs detectable by next-generation sequencing at baseline who were treatment-eligible and treated with currently recommended drugs achieved a virological response. The presence of pre-existing HBV RASs has no impact on the outcome of therapy if potent drugs with a high barrier to resistance are used.
\end{abstract}

\section{KEYWORDS}

antiviral treatment, hepatitis $B$, next-generation sequencing, primary resistance

\footnotetext{
Abbreviations: cccDNA, closed circular DNA; CHMP, Committee for Medicinal Products for Human Use; ElA, enzyme immunoassay; EMA, European Medicines Agency; HBV, hepatitis B virus; HCV, hepatitis C virus; HDV, hepatitis D virus; IQR, interquartile range; NUCs, nucleoside and nucleotide analogues; pegIFN, pegylated interferon; RASs, resistance-associated substitutions; TAF, tenofovir alafenamide.

${ }^{*}$ A complete list of the Hepatology Reference Centers and Laboratories NETWORK for chronic hepatitis B surveillance is provided in the Data S1.
} 


\section{1 | INTRODUCTION}

Chronic hepatitis B can be treated either with pegylated interferon (pegIFN) alpha, administered for a finite duration, or with nucleoside or nucleotide analogue that must most often be administered lifelong. ${ }^{1,2} \mathrm{An}$ important feature of hepatitis B virus (HBV) nucleoside and nucleotide analogues is their ability to select resistant viruses upon their long-term administration as monotherapies. Indeed, lamivudine and adefovir were shown to be associated with virological breakthroughs due to the selection of resistant viruses in $70 \%$ and $29 \%$ of patients after 5 years of administration, respectively, while telbivudine selected resistant viruses in $17 \%$ of cases after 2 years. ${ }^{1}$ In contrast, entecavir and tenofovir have a high barrier to resistance and exceptionally selected resistant viruses over up to 6-7 years of administration. ${ }^{1,3-5}$

Hepatitis B virus resistance is characterized by the on-treatment outgrowth of viral populations carrying resistance-associated substitutions (RASs) on their genome. Resistant viruses are not generated by the antiviral drugs. They pre-exist before the start of treatment, as a result of the quasispecies distribution of HBV populations and RAS archiving in covalently closed circular DNA (cccDNA) that acts as an intra-hepatic reservoir and a source for production of resistant HBV variant populations that are subsequently selected during treatment. ${ }^{6,7}$ RASs confer substantially reduced susceptibility to antiviral drugs with a low barrier to resistance, whereas drug susceptibility is poorly altered with compounds with a high barrier to resistance. The fitness of these "primary" RASs may gradually increase during therapy through the occurrence and subsequent selection of compensatory (or "secondary") substitutions, also called fitness-associated substitutions.

Because they are less fit than wild-type virus in the absence of treatment, naturally occurring resistant variants are generally undetectable at treatment baseline with classical sequencing techniques, such as population sequencing. ${ }^{8,9}$ Thus, the actual prevalence of HBV RASs and fitness-associated substitutions at baseline of nucleoside/nucleotide analogue therapy remains unknown.

The goals of this study were to use a highly sensitive sequencing method based on deep sequencing to characterize the natural prevalence of HBV RASs and fitness-associated substitutions in a large cohort of treatment-naïve patients chronically infected with HBV, to analyse the clinical and biological correlates of the presence of HBV RASs and to assess their impact on antiviral treatment responses.

\section{2 | MATERIALS AND METHODS}

\section{1 | Patients}

Santé publique France (the French national public health agency) conducted a prospective cohort study of all patients with chronic hepatitis $B$ referred for the first time to one of the 33 French reference centres between 2008 and 2012. The first 607 patients included were selected for the virological study. As shown in Figure 1, 345 patients met the selection criteria for inclusion in the present study, that is, being treatment-naïve with an HBV DNA level $\geq 2.5$ log international units (IU)/mL (minimum amount of HBV DNA for efficient PCR amplification). Among them, the amount of serum or plasma available was insufficient in 50 cases, initial PCR amplification failed in two cases and emulsion PCR failed in 61 cases. Thus, 232 patients were available for analysis (Figure 1).

The study was approved by the appropriate ethics committee. All patients gave written informed consent.

\subsection{Assessment of liver fibrosis}

The fibrosis stage was assessed by means of liver biopsy and/or transient elastography (FibroScan ${ }^{\circledR}$, Echosens, Paris, France) and/or FibroTest (BioPredictive, Paris, France). Cirrhosis was defined by a METAVIR score F4 on liver biopsy, a FibroScan score $\geq 14.6 \mathrm{kPa}$ or a FibroTest score $\geq 0.85$.

\section{3 | Laboratory measurements}

Serum HBV DNA levels were measured by means of the real-time PCR assay Cobas Ampliprep/Cobas TaqMan HBV version 2 (Roche Molecular Systems, Pleasanton, CA), according to the manufacturer's instructions. Serum HBsAg levels were quantified by means of the Architect ${ }^{\circledR} \mathrm{HBsAg}$ assay. The HBe status was determined
FIGURE 1 Patient disposition (Rx: treatment; UDPS: ultra-deep pyrosequencing; emPCR: emulsion PCR)

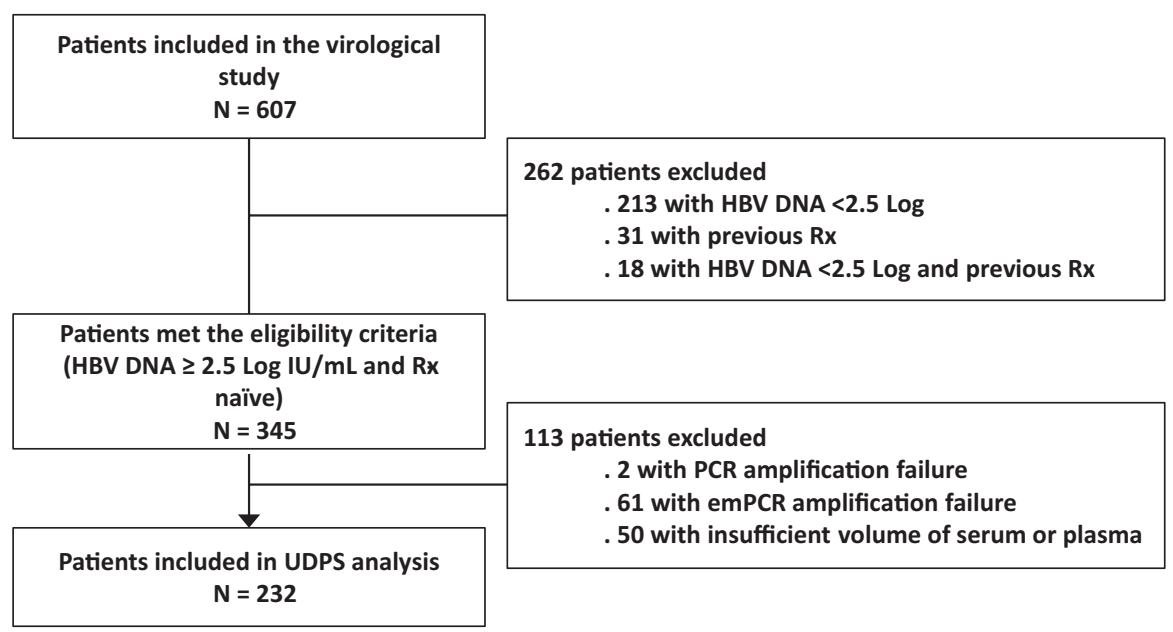


by means of a commercial enzyme immunoassay (EIA) (VIDAS ${ }^{\mathrm{TM}}$ $\mathrm{HBe} /$ Anti-HBe, Biomérieux, Marcy-l'Etoile, France). Serological markers for hepatitis $D$ virus (HDV) were assessed by means of EIA (ETI-AB-DELTAK-2, Bio-Rad Laboratories, Hercules, CA). The HBV genotype was determined by directly sequencing a portion of the overlapping genes encoding $\mathrm{HBsAg}$ and the $\mathrm{B}$ and $\mathrm{C}$ subdomains of the HBV reverse transcriptase, as previously described. ${ }^{8}$ Hepatitis $\mathrm{C}$ virus (HCV) antibodies were sought by means of the VITROS Eci/ECiQ immunodiagnostic system (Ortho-Clinical Diagnostics, Raritan, Franklin, NJ), while the Architect automated device (HIV Ag/Ab Combo, Abbott Diagnostic, Chicago, IL) was used for simultaneous detection of HIV-1 p24 antigen and HIV-1 and HIV-2 antibodies.

\subsection{Deep sequencing of the HBV reverse transcriptase region}

The nearly full-length HBV reverse transcriptase gene was PCRamplified and deep-sequenced by means of the GS FLX Titanium sequencing kit on an FLX Genome Sequencer (454 Life Sciences, Roche Diagnostics, Branford, CT), as previously described. ${ }^{9}$ The sequences generated were analysed by means of our in-house PyroPack ${ }^{\circledR}$ software package (Créteil, France). ${ }^{9}$ Briefly, the sequences were ascribed to each patient by means of identity tags and PyroClass ${ }^{\circledR}$. Then, the sequences were quality filtered and aligned, and substitutions were analysed statistically by means of PyroMute ${ }^{\circledR}$ to eliminate low-quality data and errors due to the procedure and keep only relevant sequences for final analysis. The consensus amino acid sequence was built and used as a reference to map genetic variability using PyroDyn ${ }^{\circledR}$.

The error rate of the technique at each amino acid positions was calculated using different dilutions of plasmid as described previously. ${ }^{9}$ Only sequences with frequencies above $1 \%$ were taken into account in the analysis.

\section{5 | Statistical analysis}

Results are presented as median with interquartile range [IQR] for continuous data and number (percentage) for categorical data. Factors associated with the presence of RASs were analysed by means of the Student's t test for continuous data or Fisher's exact test for categorical data.

Statistical analysis was performed with Stata ${ }^{\circledR} 11.0$ (StataCorp LP, College Station, TX). $P$ values $<0.05$ were considered as statistically significant.

\section{3 | RESULTS}

\subsection{Characteristics of the study patients}

Table 1 shows the baseline characteristics of the 232 patients included in the primary HBV resistance study. The median age was 46 years, and $61.2 \%$ of them were males. Approximately one-third of patients were born in France and another third in Sub-Saharan Africa, while $12.8 \%$ originated from Asia. Most patients (87.1\%) were $\mathrm{HBeAg}$-negative. At baseline, the mean $\mathrm{HBV}$ DNA and $\mathrm{HBsAg}$ levels were $4.12 \pm 1.65$ and $3.60 \pm 0.70 \mathrm{Log} \mathrm{IU} / \mathrm{mL}$, respectively. The most frequent HBV genotypes were A, D and E. Sixty-one of the 174 patients who had fibrosis assessment (35.1\%) had significant fibrosis, and 18 patients (10.3\%) had cirrhosis. Six patients (2.6\%) were coinfected with HDV, 2 (0.9\%) with HIV and 4 (1.8\%) with HCV.

\subsection{Natural variability of the HBV reverse transcriptase}

Figure 2 shows plots of variability per reverse transcriptase amino acid sequence position (positions 66 to 275) relative to the genotype-specific consensus sequence: $80.5 \%(169 / 210)$ of the positions had a less than $10 \%$ average amino acid variability as compared to the consensus sequence. Among the 41 remaining

TAB LE 1 Baseline characteristics of the study population $(N=232)$

\begin{tabular}{|c|c|}
\hline Age, y [median (range)] & $46(26-89)$ \\
\hline Sex, male [n (\%)] & $142(61.2)$ \\
\hline \multicolumn{2}{|l|}{ Country of birth [n (\%)] } \\
\hline France & $75(33.2)$ \\
\hline Europe, excluding France & $25(11.1)$ \\
\hline North Africa & $20(8.8)$ \\
\hline Middle East & $16(7.1)$ \\
\hline Asia & $29(12.8)$ \\
\hline Sub-Saharan Africa & $61(27.0)$ \\
\hline HDV coinfected [n (\%)] & $6(2.6)$ \\
\hline HBe-negative [n (\%)] & $202(87.1)$ \\
\hline HBV DNA level (Log IU/mL), median (IQR) & $3.5(2.9-5.1)$ \\
\hline HBsAg level (Log IU/mL), median (IQR) & $3.7(3.2-4.1)$ \\
\hline \multicolumn{2}{|l|}{ HBV genotype [n (\%)] } \\
\hline A & $84(36.2)$ \\
\hline B & $12(5.2)$ \\
\hline $\mathrm{C}$ & $18(7.8)$ \\
\hline $\mathrm{D}$ & $78(33.6)$ \\
\hline $\mathrm{E}$ & $39(16.8)$ \\
\hline G & $1(0.4)$ \\
\hline \multicolumn{2}{|l|}{ Fibrosis stage distribution [n (\%)] } \\
\hline F0-F1 & $113(48.7)$ \\
\hline $\mathrm{F} 2$ & $33(14.2)$ \\
\hline F3 & $10(4.3)$ \\
\hline $\mathrm{F} 4$ & $18(7.8)$ \\
\hline Not done & $58(25.0)$ \\
\hline ALT level (U/L) [median (IQR])(range)] & $\begin{array}{l}36(24-56) \\
(8-496)\end{array}$ \\
\hline ALT > ULN [n (\%)] & $86(37.1)$ \\
\hline
\end{tabular}


positions, 17 had a variability comprised between $10 \%$ and $20 \%$, 16 between $20 \%$ and $30 \%$, five between $30 \%$ and $40 \%$, and three between $40 \%$ and $50 \%$ (Table 2). Three hot spots of variability, spanning amino acid positions 121-153, 211-224 and 256-273 were identified outside of reverse transcriptase domains $A$ to $E$. These results indicate that $\mathrm{HBV}$ reverse transcriptase sequences are conserved across $\mathrm{HBV}$ isolates and quasispecies variants, regardless of the HBV genotype.

\section{3 | Natural variability of $\mathrm{HBsAg}$}

The same sequence data set was analysed in the alternative open reading frame coding for $\mathrm{HBsAg}$. Figure 3 shows plots of variability per HBsAg amino acid sequence position (positions 107 to 226) according to the HBV genotype. Overall, $80.8 \%$ (97/120) of the positions had an average variability of less than $10 \%$ as compared to the genotype-specific consensus sequence. Among the 23 remaining positions, 12 had a variability comprised between $10 \%$ and $20 \%$, nine between $20 \%$ and $30 \%$, and two between $30 \%$ and $40 \%$. Of the 23 variable $\mathrm{HBsAg}$ positions, six (positions 126 , $127,131,134,140$ and 143) resided within the "a" determinant of HBsAg major hydrophobic region (amino acids 124-147). Among them, all but two (positions 126 and 140) have already been described to be polymorphic (http://hbv.bioinf.mpi-inf.mpg.de/ index.php). Amino acid changes at position 126 were reported to be associated with active or passive immunization escape. ${ }^{10}$ No differences were observed between the different HBV genotypes (Figure 3).

\section{4 | Prevalence of HBV RASs and fitness- associated substitutions}

Among the 232 treatment-naïve patients, 58 (25.0\%) had detectable RASs in their serum or plasma by deep sequencing with a cut-off of $1 \%$. They represented $1 \%-5 \%$ of the viral quasispecies in 43 of 232 patients (18.5\%), 5\%-10\% in 5 of 232 patients (2.2\%), $10 \%-20 \%$ in 6 of 232 patients (2.6\%) and $>20 \%$ in 4 of 232 patients (1.7\%). Among these 58 patients, 41 (70.7\%) had only RASs, whereas the remaining 17 (29.3\%) had fitness-associated substitutions in addition to their RASs. Finally, 53 additional patients (22.8\%) had detectable fitness-associated substitutions not associated with RASs (Figure 4).

With a cut-off of $1 \%$, RASs or fitness-associated substitutions were found at all amino acid positions known to be associated with resistance to currently approved nucleoside/nucleotide analogues or with increased fitness of resistant variants (including amino acid positions 163, 169, 173, 180, 181, 184, 186, 202, 204, 236 and 250 ), with the exception of position 80 (Table 3). Only five positions (rtl163, rtL180, rtA181, rtR202 and rtM204) harboured substitutions present in $>20 \%$ of the patients' HBV variant populations. Substitutions at other positions were less prevalent in the quasispecies when present (Table 3).
HBV genotype A $(n=84)$

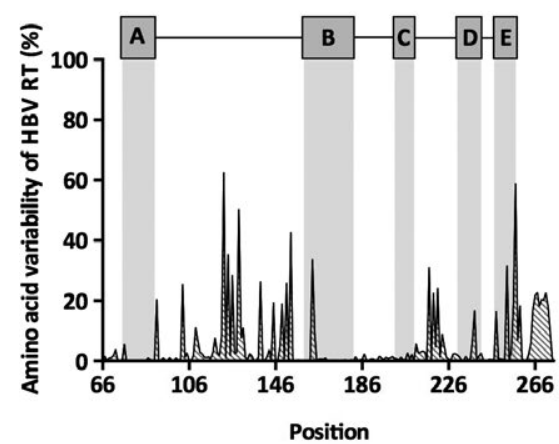

HBV genotype D $(\mathbf{n}=\mathbf{7 8})$

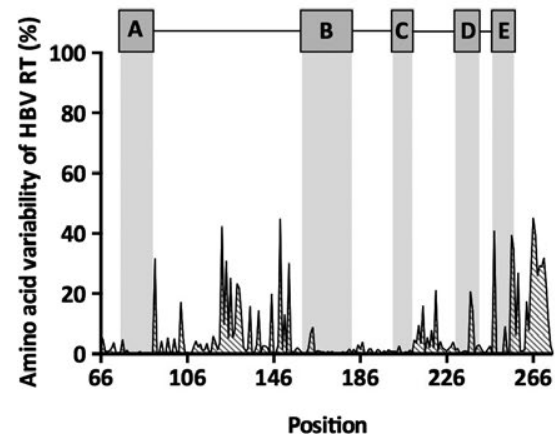

HBV genotype B $(n=12)$

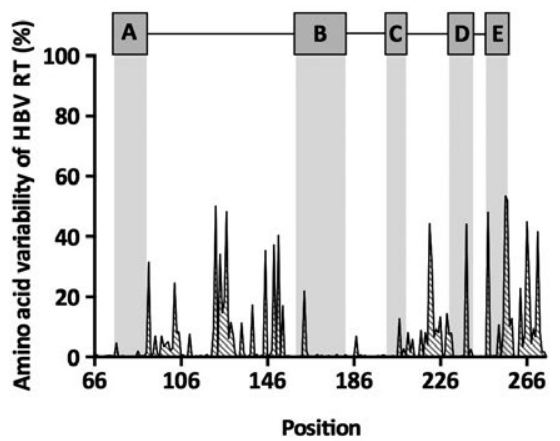

HBV genotype $E(n=39)$

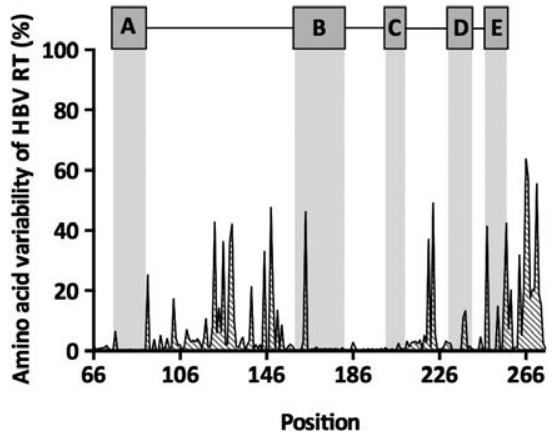

HBV genotype $C(n=18)$

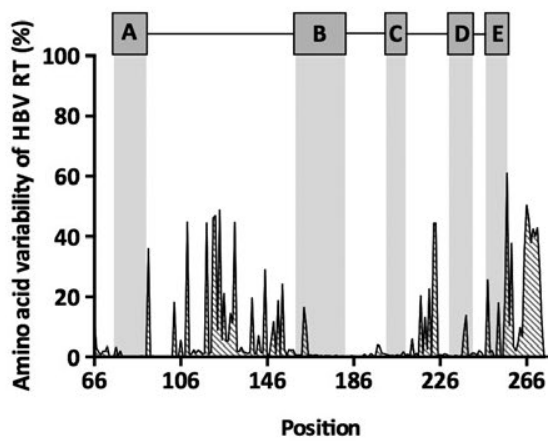

FIG URE 2 Natural amino acid variability of the HBV RT (amino acid positions 66 to 275) in 232 treatment-naïve patients chronically infected with HBV genotype $A(n=84)$, genotype $B(n=12)$, genotype $C(n=18)$, genotype $D(n=78)$ and genotype $E(n=39)$. Functional domains A (aa 75-90), B (aa 159-182), C (aa 201-210), D (aa 230-241) and E (aa 247-257) of the HBV RT are shown in grey 


\begin{tabular}{|l|l|}
\hline Degree of variability & Amino acid positions \\
\hline $10 \%-20 \%$ & S109; N118; I121; G127; Q130; N131; N139; Y148; I163; L164; \\
& L217; S219; V224; V253; D263; C272; F273 \\
\hline $20 \%-30 \%$ & L91; V103; H126; M129; L145; Q149; F151; R153; F221; A223; \\
& H238; S259; I265; K268; I269; K270 \\
\hline $30 \%-40 \%$ & H124; H248; C256; Q267; E271 \\
\hline $40 \%-50 \%$ & L122; Y257; I266 \\
\hline
\end{tabular}

TAB LE 2 Natural variability of the HBV reverse transcriptase: the degree of variability is indicated for specific HBV reverse transcriptase positions
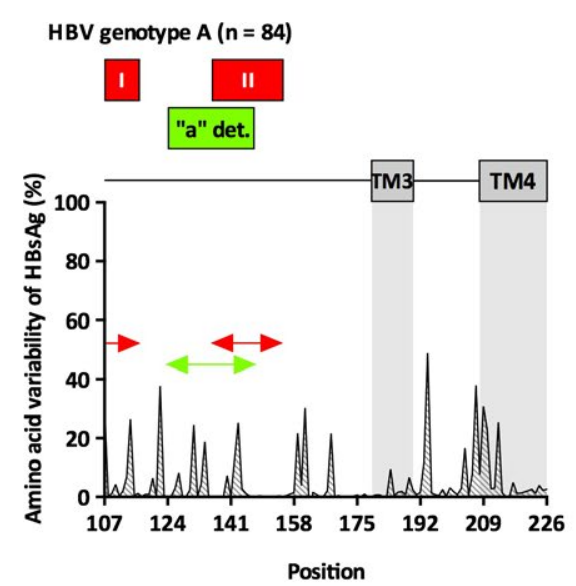

HBV genotype $D(n=78)$

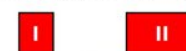

"a" det.

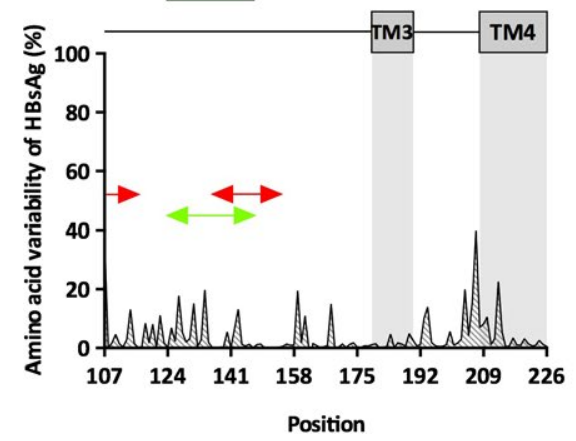

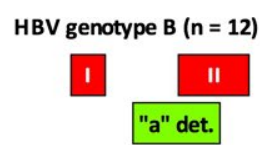

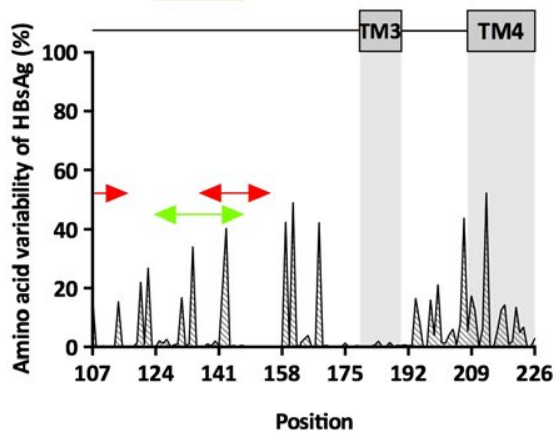

HBV genotype $E(n=39)$

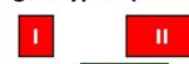

"a" det.

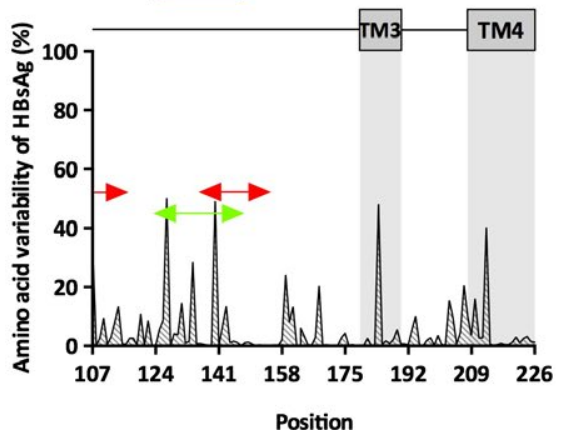

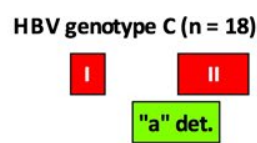

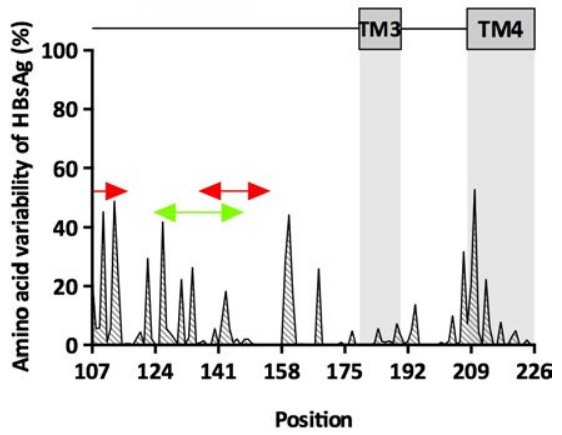

FIGURE 3 Natural amino acid variability of HBsAg (amino acid positions 107 to 226) in 232 treatment-naïve patients chronically infected with HBV genotype $A(n=84)$, genotype $B(n=12)$, genotype $C(n=18)$, genotype $D(n=78)$ and genotype $E(n=39)$. The positions of HLA I epitope (I, aa 97-107) and HLA II epitope (II, aa 163-174) are shown in red. HBsAg "a" determinant (aa 124-147), the main B cell epitope, is shown in green. Transmembrane domains 3 (TM3, aa 179-190) and 4 (TM4, aa 208-226) are represented in grey

\subsection{HBV resistance profiles}

Table 4 shows the individual profiles of RASs and/or fitnessassociated substitutions in the 58 patients with detectable RASs by deep sequencing. $\mathrm{rtM} 204 \mathrm{I} / \mathrm{V} \pm \mathrm{rtL} 180 \mathrm{M}$ were present in 22 of the 58 patients in proportions ranging from $1.2 \%$ to $22 \%$ of the viral quasispecies. In most cases, the resistance profiles were complex, with the presence of multiple additional substitutions at positions rt163, rt173, rt202, rt250 and/or rt181 (Table 3). Of the 58 patients, only four displayed an entecavir resistance profile, characterized by the presence of substitutions at positions 202 and 250 in addition to rtM204I/V, present in small proportions ( $1.3 \%$ to $4.3 \%$ of the quasispecies) in all cases. A RAS at position rt186 was detected in four patients, alone or in combination with other substitutions at positions rt184, rt181 and/ or rt236, always present in low proportions. In addition, substitutions at positions $\mathrm{rt169}$, rt184, rt202 or rt250 were detected in 21 patients without RASs at position rt204, in proportions ranging from $1 \%$ to $22.9 \%$ of the quasispecies. rtl163V, which increases the fitness of rtA186T-carrying variants in the presence of entecavir, ${ }^{11}$ was detectable in 67 of 232 treatment-naïve patients (28.9\%), generally in high proportions (over $20 \%$ of the quasispecies in 43 of them). However,

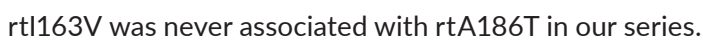

Of the 58 patients with detectable RASs, 19 harboured an adefovir resistance profile, including the presence of $\mathrm{rtA} 181 \mathrm{~V} / \mathrm{T}$ in $14 \mathrm{pa}-$ tients, rtN236T in four patients and both in one patient. rtA181V/T was generally present in low proportion, except in three patients in 
whom it represented $13.0 \%, 13.8 \%$ and $85.2 \%$ of the viral quasispecies, respectively. rtN236T was also present in low proportion (<5\%) in all but one case. In all patients but 4 , adefovir RASs were detected

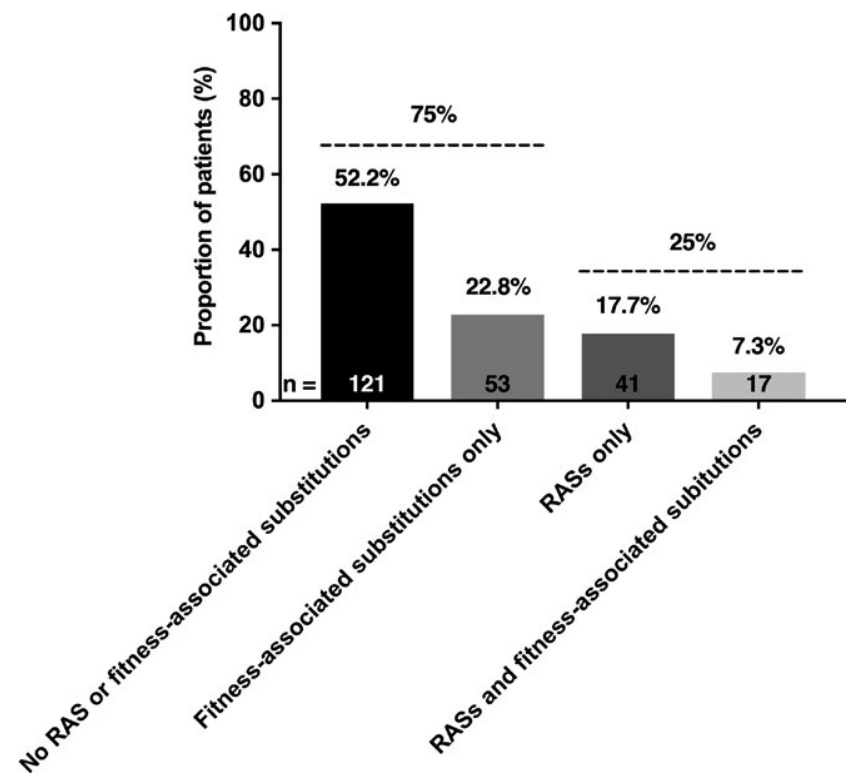

FIGURE 4 Proportion of treatment-naïve patients with and without detectable RASs or fitness-associated substitutions $(n=232)$ in combination with other substitutions at positions $163,173,180$, 184, 186 and/or 204.

\subsection{Factors associated with the presence of HBV RASs and fitness-associated substitutions}

The age, gender, country of birth, HBV genotype, ALT level and fibrosis stage were not associated with the presence of one or several substitutions (including RASs and fitness-associated substitutions). In contrast, the HBsAg level was significantly higher in patients with at least one detectable substitution than in those without any substitution $(3.7 \pm 0.7 \mathrm{Log} \mathrm{IU} / \mathrm{mL}$ versus $3.5 \pm 0.7 \mathrm{Log} \mathrm{IU} / \mathrm{mL}$; $P=0.047$ ). In addition, the HBV DNA level was significantly higher in patients with at least two substitutions, as compared to those without any substitution $(5.4 \pm 1.8 \mathrm{Log} \mathrm{IU} / \mathrm{mL}$ vs $4.0 \pm 1.7 \mathrm{Log} \mathrm{IU} /$ $\mathrm{mL} ; P<0.001$ ). The country of birth (Europe, North Africa and Asia; $P<0.001$ ) and the HBV genotype (genotypes A, $B$ and $C ; P=0.017$ ) were also significantly associated with the presence of the rtl163V RAS, whereas an HDV coinfection was significantly associated with the presence of substitutions at position $\mathrm{rt181}(P=0.044)$.

\section{7 | Influence of baseline RASs on subsequent treatment response}

Among the patients who had detectable RASs at baseline and were eligible to antiviral treatment, 22 were treated. Treatment was based
TABLE 3 Proportions of treatmentnaïve patients with various amounts of detectable resistance-associated substitutions (RASs) or fitness-associated substitutions at amino acid positions of the HBV RT known to harbour such substitutions

\begin{tabular}{llllll} 
& \multicolumn{5}{l}{$\begin{array}{l}\text { Distribution of RASs and/or fitness-associated substitution } \\
\text { frequencies in the viral quasispecies (\%) }\end{array}$} \\
\cline { 2 - 6 } $\begin{array}{l}\text { RASs or fitness-associated } \\
\text { substitutions }\end{array}$ & $<1 \%$ & $1 \%-5 \%$ & $5 \%-10 \%$ & $10 \%-20 \%$ & $>20 \%$ \\
\hline rtL80V & 100 & 0 & 0 & 0 & 0 \\
\hline rtL80I & 100 & 0 & 0 & 0 & 0 \\
\hline rt163V & 71.1 & 5.6 & 2.2 & 2.6 & 18.5 \\
\hline rt169T & 98.7 & 0.9 & 0 & 0.4 & 0 \\
\hline rtV173L & 97.8 & 1.3 & 0.4 & 0.4 & 0 \\
\hline rtL180M & 98.7 & 0.9 & 0 & 0 & 0.4 \\
\hline rtA181V & 98.3 & 1.3 & 0 & 0 & 0.4 \\
\hline rtA181T & 94.8 & 3.0 & 1.3 & 0.9 & 0 \\
\hline rtT184S & 98.7 & 0.9 & 0.4 & 0 & 0 \\
\hline rtT184A & 98.7 & 1.3 & 0 & 0 & 0 \\
\hline rtT184I & 100 & 0 & 0 & 0 & 0 \\
\hline rtT184L & 100 & 0 & 0 & 0 & 0 \\
\hline rtT184G & 100 & 0 & 0 & 0 & 0 \\
\hline rtA186T & 98.3 & 1.7 & 0 & 0 & 0 \\
\hline rtR202G & 96.1 & 3.5 & 0 & 0 & 0.4 \\
\hline rtR202I & 99.1 & 0 & 0.9 & 0 & 0 \\
\hline rtM204V & 95.7 & 3.0 & 0.4 & 0 & 0.9 \\
\hline rtM204I & 94.8 & 4.3 & 0.9 & 0 & 0 \\
\hline rtN236T & 97.8 & 1.7 & 0 & 0.4 & 0.4 \\
\hline rtM250V & 3.9 & 0 & 0 & 0 \\
\hline
\end{tabular}


TAB LE 4 Profiles of resistance-associated substitutions and/or fitness-associated substitutions observed in 58 treatment-naïve patients by means of deep sequencing

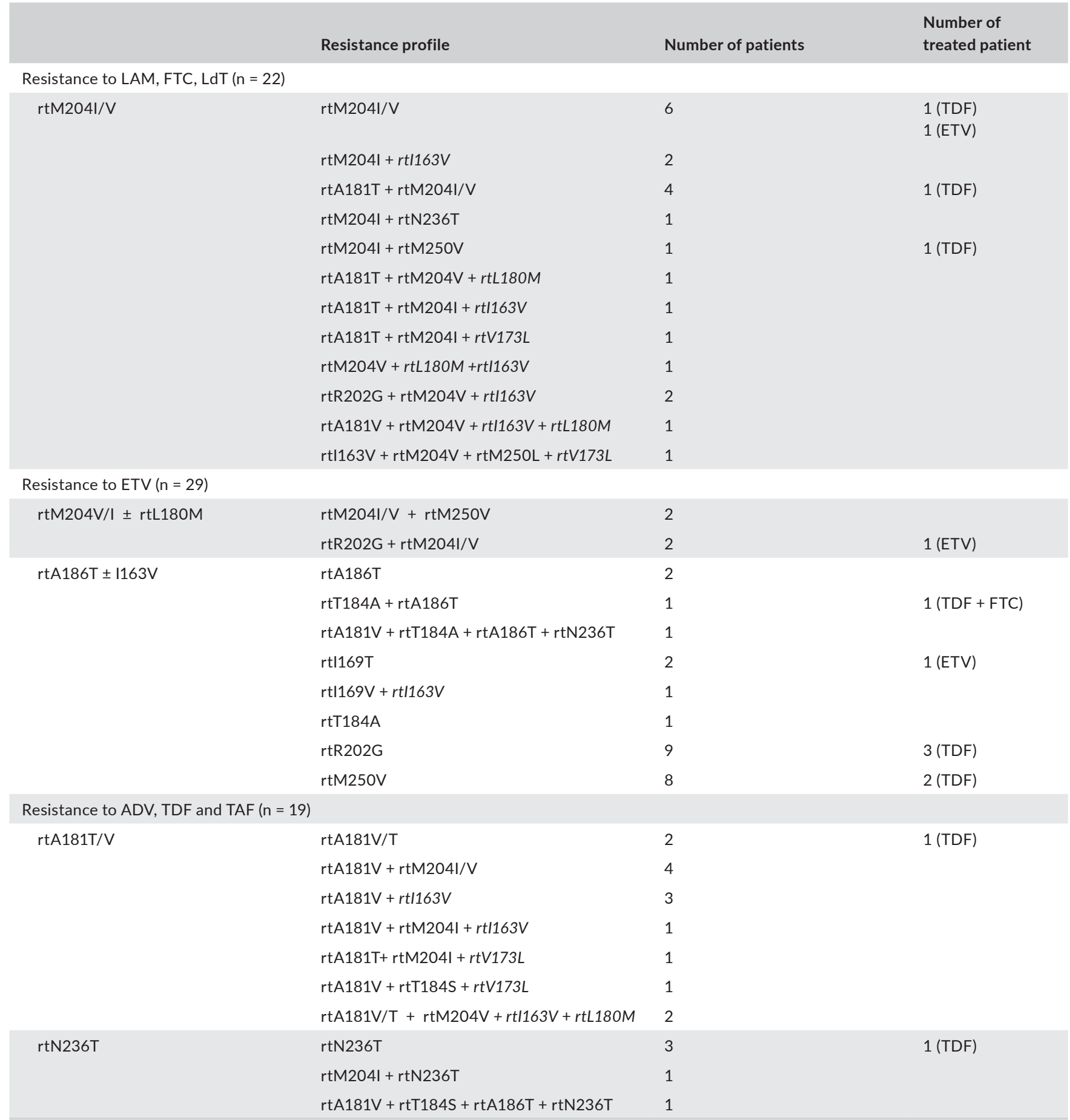

rtM204I/V confer resistance to lamivudine (LAM), emtricitabine (FTC) and telbivudine (LdT), and reduced susceptibility to entecavir (ETV), while rtL180M improves the fitness of rtM204V-carrying variants.

Together with rtM204 \pm rtL180, substitutions at positions rt169, rt184, rt202 or rt250 increase the level of resistance to ETV.

rtA186T is associated with ETV resistance, and substitutions at position rt163 improve the fitness of the corresponding variants. ${ }^{11}$

rtA181V/T and rtN236T confer reduced susceptibility to adefovir (ADV) and, to a lesser extent, to tenofovir or tenofovir alafenamide (TDF or TAF), at least in vitro.

rtA181V/T also confer reduced susceptibility to LAM and LdT. Fitness-associated substitutions are in italic.

on pegylated interferon in three cases and on nucleoside/nucleotide analogues in 19 cases, including lamivudine in one patient, entecavir in six patients and tenofovir in 12 patients (in combination with emtricitabine in one case). A virological response, defined as an undetectable HBV DNA 48-96 weeks after the start of treatment, was achieved in the 14 patients receiving a nucleoside/nucleotide 
analogue regimen for whom follow-up was available (two patients receiving entecavir, two patients receiving tenofovir and one patient receiving lamivudine were lost to follow-up). The three IFN-treated patients all had undetectable HBV DNA 48 months after the start of treatment.

\section{DISCUSSION}

Drug resistance is the principal cause of treatment failure in patients treated with nucleoside or nucleotide analogues who are adherent. However, little information is available on the prevalence of resistant HBV variants in treatment-naïve patients. Classical techniques, such as population sequencing, reverse DNA hybridization, restriction fragment length polymorphism or cloning of PCR products followed by sequencing of the clones, have been widely used for HBV resistance testing in both in vitro and in vivo research studies. ${ }^{12,13}$ These molecular methods have limitations relating to their specificity, sensitivity, ability to detect novel mutations and reporting of qualitative results. Population sequencing- and reverse hybridization-based assays are commercially available and have been used in clinical practice. ${ }^{14,15}$ These methods are useful to identify highly prevalent resistant populations in patients who failed on therapy. In contrast, population sequencing is not sensitive enough to detect minor populations because of their poor fitness compared to wild-type viruses, while hybridization-based assays are unable to detect unknown RASs. ${ }^{16}$ Next-generation sequencing (NGS) techniques based on deep sequencing were recently developed to increase the sensitivity and accuracy of classical techniques. They are highly sensitive to detect minor viral populations and well-suited to viral resistance studies. Despite the potential clinical utility of NGS in the monitoring of resistance during antiviral therapy, there remain barriers to its use, including high cost, the lack of molecular expertise and bioinformatics/statistical capabilities. ${ }^{12,17}$ However, NGS-based techniques provide invaluable tools for molecular studies of viral resistance.

In the present study, we used a sensitive NGS-based approach to show that HBV RASs pre-exist in a substantial proportion ( 25\%) of patients with chronic HBV infection never exposed to nucleoside/nucleotide analogues. This proportion was higher in our study using NGS than in previous works using less sensitive sequencing methods, ${ }^{18}$ and in the same order as in another study using NGS with a cut-off of $1 \%$ to longitudinally monitor a small number of treated patients. ${ }^{19}$ All positions associated with reduced susceptibility to nucleoside/nucleotide analogues were found to carry substitutions in one to several cases in our study. RASs were generally present in low proportion within the viral quasispecies, a finding in keeping with their reduced fitness in the absence of drug administration. However, RASs at positions 181 and 204 were frequently found (15 and 22 patients, respectively) and were present in moderate to high proportions, explaining the high incidence of virological breakthroughs due to the selection of resistant HBV variants when low-barrier to resistance drugs, such as lamivudine, adefovir or telbivudine, are used.
Currently, these compounds are no longer recommended as firstline treatments for hepatitis B in international guidelines. Instead, compounds with a high barrier to resistance should be used, including entecavir or tenofovir. ${ }^{1,2}$ In our study, most of the patients with RASs detectable at baseline who were treatment-eligible were treated with entecavir, tenofovir, the combination of tenofovir and emtricitabine or pegylated IFN. All of them experienced a virological response characterized by an undetectable HBV DNA 48-96 weeks after the start of antiviral treatment. Thus, our study shows that the presence of pre-existing HBV RASs has no impact on the outcome of therapy if potent drugs with a high barrier to resistance are used, at least on the mid-term.

Our study has a few limitations. First, the least frequent genotypes (G, H and I) were not represented. Secondly, the rate of deep sequencing failure was high, in the order of $20 \%$, similar to what has been observed in other studies with the method used in the present study. ${ }^{20}$ However, no difference was found on the other parameters between these patients and those whose deep sequencing was successful.

Only few baseline parameters were associated with the presence of RASs. Not surprisingly, a higher HBV DNA level was associated with a greater HBV variability. The relationship with the $\mathrm{HBsAg}$ level is difficult to explain and suggests a confounding factor, such as the genotype, although their relationship was not found in our series, possibly because the number of patients was too small in the different genotype groups. A relationship was found between the presence of certain RASs at baseline and the country of birth, that could also reflect a role for the HVB genotype, the route of transmission and/or the duration of infection. A relationship with the HDV status was also found, which could also be explained by different epidemiologies.

In conclusion, our study showed that HBV RASs that confer reduced susceptibility to nucleoside/nucleotide analogues are frequently found in patients who have never been exposed to these compounds when a highly sensitive method, with a limit of detection of $1 \%$, is used. It is likely that, if even more sensitive techniques with a detection cut-off far below $1 \%$ were used, RASs and fitness-associated substitutions would be found in nearly all patients before starting antiviral treatment. Although RASs were generally present in low proportions in the viral quasispecies, those conferring resistance to lamivudine, adefovir or telbivudine were often found in high proportions, as a result of their good fitness as compared to wild-type viruses. This provides a theoretical basis to support the use of nucleoside/nucleotide analogues with a high barrier to resistance, such as entecavir or tenofovir, as first-line therapy, as recommended by international guidelines. ${ }^{1,2}$ The drug patents expired in 2015 for entecavir, 2017 for tenofovir. Generic drugs for both entecavir and tenofovir disoproxil fumarate have been approved by the FDA and can thus be used in many other parts of the world, allowing for improved access to care. In addition, tenofovir alafenamide (TAF) has been approved for the treatment of chronic HBV infection with compensated liver disease by the FDA in the US and has received a positive opinion 
from the Committee for Medicinal Products for Human Use, the scientific committee of the European Medicines Agency. This prodrug of tenofovir has demonstrated similar antiviral efficacy with improved renal and bone safety as compared to TDF, due to greater plasma stability and more efficient delivery of tenofovir to hepatocytes. ${ }^{21}$ Thus, in spite of our findings showing a high prevalence of HBV RASs at baseline in patients never treated with nucleoside/nucleotide analogues, it is possible to broadly achieve high rates of virological response, provided that the cascade of care is optimized.

\section{ACKNOWLEDGEMENTS}

We would like to thank the patients and nurses for their involvement in the study, as well as the French Society of Hepatology (Association Française pour l'Étude du Foie, AFEF), the Fédération Nationale des Pôles de Référence et Réseau Hépatites, the National Agency for Research on AIDS and Viral Hepatitis (ANRS), and all the clinicians and virologists from the reference hepatology centers and laboratories (listed in Data S1).

\section{CONFLICT OF INTERESTS}

S.C. has received research grants from Gilead and has served as an advisor and/or speaker for Abbott, AbbVie, Bristol-Myers Squibb, Cepheid, Gilead, Hologic and Merck. F.Z. has received research grants from Gilead and has served as an advisor and/or speaker for Gliead and Bristol-Myers Squibb. J.-M.P. has received research grants from Abbott, AbbVie and Gilead and has served as an advisor and speaker for Abbott, Siemens, AbbVie, Gilead and Merck. Other authors have no conflict of interests to disclose.

\section{ORCID}

Stéphane Chevaliez iD http://orcid.org/0000-0001-6913-9017

Fabien Zoulim (iD http://orcid.org/0000-0002-2245-0083

\section{REFERENCES}

1. European Association for the Study of the Liver. EASL 2017 Clinical Practice Guidelines on the management of hepatitis B virus infection. J Hepatol. 2017;67(2):370-398.

2. Terrault NA, Lok AS, McMahon BJ, et al. Update on prevention, diagnosis, and treatment and of chronic hepatitis B: AASLD 2018 Hepatitis B Guidance. Hepatology. 2018;16:1560-1599.

3. Buti M, Tsai N, Petersen J, et al. Seven-year efficacy and safety of treatment with tenofovir disoproxil fumarate for chronic hepatitis $B$ virus infection. Dig Dis Sci. 2015;60(5):1457-1464.

4. Kitrinos KM, Corsa A, Liu Y, et al. No detectable resistance to tenofovir disoproxil fumarate after 6 years of therapy in patients with chronic hepatitis B. Hepatology. 2014;59(2):434-442.

5. Liu Y, Corsa AC, Buti M, et al. No detectable resistance to tenofovir disoproxil fumarate in $\mathrm{HBeAg}+$ and $\mathrm{HBeAg}$ - patients with chronic hepatitis B after 8 years of treatment. J Viral Hepat. 2017;24(1):68-74.
6. Pawlotsky JM. The concept of hepatitis B virus mutant escape. J Clin Virol. 2005;34(Suppl 1):S125-S129.

7. Zoulim F, Locarnini S. Hepatitis B virus resistance to nucleos(t)ide analogues. Gastroenterology. 2009;137(5):1593-1608. e1591-1592.

8. Pallier C, Castera L, Soulier A, et al. Dynamics of hepatitis B virus resistance to lamivudine. J Virol. 2006;80(2):643-653.

9. Rodriguez C, Chevaliez S, Bensadoun P, Pawlotsky JM. Characterization of the dynamics of hepatitis $B$ virus resistance to adefovir by ultra-deep pyrosequencing. Hepatology. 2013;58(3):890-901.

10. Wallace LA, Carman WF. Surface gene variation of HBV: scientific and medical relavance. Vir Hep Rev. 1997;3:5-16.

11. Hayashi S, Murakami S, Omagari K, et al. Characterization of novel entecavir resistance mutations. J Hepatol. 2015;63(3):546-553.

12. Chevaliez S, Rodriguez C, Pawlotsky JM. New virologic tools for management of chronic hepatitis B and C. Gastroenterology. 2012;142(6):1303-1313. e1301.

13. Rybicka M, Stalke P, Bielawski KP. Current molecular methods for the detection of hepatitis B virus quasispecies. Rev Med Virol. 2016;26(5):369-381.

14. Degertekin B, Hussain M, Tan J, Oberhelman K, Lok AS. Sensitivity and accuracy of an updated line probe assay (HBV DR vol 3) in detecting mutations associated with hepatitis $B$ antiviral resistance. J Hepatol. 2009;50(1):42-48.

15. Huh HJ, Kim JY, Lee MK, Lee NY, Kim JW, Ki CS. Analytical and clinical evaluation of the Abbott RealTime hepatitis $B$ sequencing assay. J Clin Virol. 2016;85:27-30.

16. Lowe CF, Merrick L, Harrigan PR, Mazzulli T, Sherlock CH, Ritchie G. Implementation of Next-Generation Sequencing for Hepatitis B Virus Resistance Testing and Genotyping in a Clinical Microbiology Laboratory. J Clin Microbiol. 2016;54(1):127-133.

17. Gargis AS, Kalman L, Berry MW, et al. Assuring the quality of next-generation sequencing in clinical laboratory practice. Nat Biotechnol. 2012;30(11):1033-1036.

18. Li X, Liu Y, Zhao $P$, et al. Investigation into drug-resistant mutations of HBV from 845 nucleoside/nucleotide analogue-naive Chinese patients with chronic HBV infection. Antivir Ther. 2015;20(2):141-147.

19. Jones LR, Sede M, Manrique JM, Quarleri J. Hepatitis B virus resistance substitutions: long-term analysis by next-generation sequencing. Arch Virol. 2016;161(10):2885-2891.

20. Audsley J, Bent SJ, Littlejohn M, et al. Effects of long-term tenofovir-based combination antiretroviral therapy in HIVhepatitis $B$ virus coinfection on persistent hepatitis $B$ virus viremia and the role of hepatitis $B$ virus quasispecies diversity. AIDS. 2016;30(10):1597-1606.

21. Murakami E, Wang T, Park Y, et al. Implications of efficient hepatic delivery by tenofovir alafenamide (GS-7340) for hepatitis B virus therapy. Antimicrob Agents Chemother. 2015;59(6):3563-3569.

\section{SUPPORTING INFORMATION}

Additional supporting information may be found online in the Supporting Information section at the end of the article.

How to cite this article: Chevaliez S, Rodriguez C, Poiteau L, et al. Primary resistance of hepatitis $B$ virus to nucleoside and nucleotide analogues. J Viral Hepat. 2019;26:278-286. https:// doi.org/10.1111/jvh.13025 